Edited by Kiriakos Xenitidis and
Colin Campbell
Contents
- Acceptance, grief and meaning
- Taking an internet history
- Dementia: suicide by drowning
- Adolescent-onset anorexia nervosa - missing
- Fallacies in standardised mortality ratios in
anorexia nervosa
- Suicide rates in people of South Asian origin in
England and Wales
- Risperidone for adolescent schizophrenia
- Time to change concepts and terminology
- Abortion and mental health disorders

\section{Acceptance, grief and meaning}

Prigerson \& Maciejewski ${ }^{1}$ assert that the resolution of grief coincides with increasing acceptance of loss, mainly cognitive and emotional acceptance. The role of spiritual acceptance has not been mentioned directly, although experiences like inner peace, tranquility and letting go, or regaining what is lost or being taken away, are more spiritual rather than emotional or intellectual. Moreover, some of the features which can be considered spiritual are included as criteria for prolonged grief disorder, ${ }^{2}$ such as confusion about one's identity and feeling that life is empty and meaningless since the loss. Issues related to culture and the meaning and value of death ${ }^{3}$ are relevant to both grief and acceptance, and I wonder whether these should also be considered.

Patients diagnosed with terminal cancer often confront existential issues. Experiences with patients with advanced or terminal cancers indicate that not only is cognitive and emotional acceptance essential, but that spiritual aspects are equally important. Spiritual acceptance of grief will help the grieved to understand the meaning and purpose of the loss. As Frankl ${ }^{4}$ states 'suffering ceases to be a suffering as soon as it finds a meaning. Longitudinal studies should clarify not only the way in which grief resolution relates to acceptance of dying and death, but also whether grief relates differentially to cognitive, emotional and spiritual acceptance. Prigerson \& Maciejewski ${ }^{1}$ conclude that decline in grief-related distress appears to correspond with an increase in peaceful acceptance of loss, which I feel could be enhanced by addressing issues related to purpose and meaning of the loss.

There is some small change besides the two sides of the coin!

1 Prigerson HG, Maciejewski PK. Grief and acceptance as opposite sides of the same coin: setting a research agenda to study peaceful acceptance of loss. Br J Psychiatry 2008; 193: 435-7.

2 Prigerson HG, Vanderwerker LC, Maciejewski PK. Prolonged grief disorder: a case for inclusion in DSM-V. In Handbook of Bereavement Research and Practice: 21st Century Perspectives (eds M Stroebe, R Hansson, H Schut, et al): 165-86. American Psychological Association Press, 2008.

3 Byock I. The meaning and value of death. J Palliat Med 2002; 5: 279-88.
4 Frankl VE. Man's Search for Meaning (4th edn). Washington Square Press, 1985.

Santosh K. Chaturvedi, National Institute of Mental Health \& Neurosciences, Bangalore, India. Email: chatur@nimhans.kar.nic.in

doi: 10.1192/bjp.194.6.561

Authors' reply: We thank Dr Chaturvedi for highlighting the potentially important role that spirituality plays in the acceptance of loss. Recent research attests to the powerful influence of spirituality and religious beliefs in shaping patients' cognitive acceptance of terminal illness, treatment preferences, and even in determining the receipt of intensive, life-prolonging care in the last week of life. ${ }^{1}$

Nevertheless, we wish to differentiate between components of grief (e.g. yearning) and factors affecting the intensity and course of grief (e.g. spirituality). We posit that grief is on the same continuum as emotional acceptance - opposite poles of a unitary dimension. We contend that both spirituality and cognitive acceptance are distinct from, but related to, emotional acceptance and grief. Spirituality might foster emotional acceptance; cognitive acceptance might exacerbate grief. Identifying factors affecting grief and emotional acceptance may suggest ways to enhance an individual's mental health and well-being in the face of death, and offer ways to minimise loss-related suffering.

As a further distinction, we consider the loss of meaning in the context of prolonged grief disorder ${ }^{2}$ to represent the emptiness experienced by the absence of an attachment figure. It is not intended to refer to a broader existential crisis. The sense of emptiness felt in grief may well lead a person to question the meaning of life. It may heighten an individual's sense of anomie (i.e. a feeling of disorientation and alienation from society caused by the perceived absence of a supporting social or moral framework) and affect a person's will to live. The meaning derived from spiritual beliefs may buffer individuals from the emptiness that follows a major interpersonal loss. Still, we do not consider spiritual beliefs to be components of grief. Rather spirituality may be a powerful antidote (perhaps, social support and social integration are others) to the pain of grief and elixir promoting emotional acceptance.

1 Phelps AC, Maciejewski PK, Nilsson M, Balboni TA, Wright AA, Paulk ME, et al. Coping with cancer: religious coping predicts use of intensive life-prolonging care near death. JAMA 2009; 301: 1140-7.

2 Prigerson HG, Vanderwerker LC, Maciejewski PK. Prolonged grief disorder: a case for inclusion in DSM-V. In Handbook of Bereavement Research and Practice: 21st Century Perspectives (eds M Stroebe, R Hansson, H Schut, et al): 165-86. American Psychological Association Press, 2008.

Holly G. Prigerson, Paul K. Maciejewski Brigham and Women's Hospital, Harvard Medical School, and Statistics Core of the Center for Psycho-oncology \& Palliative Care Research, Dana-Farber Cancer Institute, 44 Binney Street, Smith 268, Boston, MA 02115, USA. Email: Holly_Prigerson@dfci.harvard.edu

doi: 10.1192/bjp.194.6.561a

\section{Taking an internet history}

Cooney \& Morris ${ }^{1}$ argue that we should consider taking an 'internet history' to help assess young people's risk of self-harm, suicide and presumed psychopathologies such as 'internet addiction'. Although an understanding of how a client uses the internet may be important, the authors caricature what we know about the risks of the internet.

Although information on suicide methods is available online, ${ }^{2}$ there is currently no clear evidence that the risk of self-harm or 